\title{
Photocatalytic Material-Microbe Hybrids: Applications in Environmental Remediations
}

\author{
Yadong Yu ${ }^{1 \dagger}$, Shanshan Wang ${ }^{1 \dagger}$, Jinrui Teng ${ }^{2}$, Anze Zupanic ${ }^{3}$, Shuxian Guo ${ }^{4}$, Xiaobin Tang ${ }^{5 *}$ \\ and Heng Liang ${ }^{5 *}$
}

${ }^{1}$ College of Biotechnology and Pharmaceutical Engineering, Nanjing Tech University, Nanjing, China, ${ }^{2} 2011$ College, Nanjing Tech University, Nanjing, China, ${ }^{3}$ Department of Biotechnology and Systems Biology, National Institute of Biology, Ljubljana, Slovenia, ${ }^{4}$ Henan Key Laboratory of Industrial Microbial Resources and Fermentation Technology, Nanyang Institute of Technology, Nanyang, China, ${ }^{5}$ State Key Laboratory of Urban Water Resource and Environment, Harbin Institute of Technology, Harbin, China

OPEN ACCESS

Edited by:

Bo Wang,

Shenzhen Institutes of Advanced

Technology (CAS), China

Reviewed by:

Xin Tian,

Soochow University, China

${ }^{*}$ Correspondence:

Xiaobin Tang

tang5462@163.com

Heng Liang

hitliangheng@163.com

${ }^{t}$ These authors have contributed equally to this work

Specialty section: This article was submitted to

Synthetic Biology,

a section of the journal Frontiers in Bioengineering and

Biotechnology

Received: 15 November 2021 Accepted: 16 December 2021 Published: 31 January 2022

Citation:

Yu Y, Wang S, Teng J, Zupanic A, Guo S, Tang $X$ and Liang $H$ (2022)

Photocatalytic Material-Microbe Hybrids: Applications in

Environmental Remediations.

Front. Bioeng. Biotechnol. 9:815181.

doi: 10.3389/fbioe.2021.815181
Environmental pollution has become one of the most urgent global issues that we have to face now. Searching new technologies to solve environmental issues is of great significance. By intimately coupling photocatalytic materials with microbes, the emerging photocatalytic material-microbe hybrid $(\mathrm{PMH})$ system takes advantages of the high-efficiency, broad-spectrum light capture capability of the photocatalytic material and the selectivity of microbial enzymatic catalysis to efficiently convert solar energy into chemical energy. The $\mathrm{PMH}$ system is originally applied for the solar-to-chemical production. Interestingly, recent studies demonstrate that this system also has great potential in treating environmental contaminations. The photogenerated electrons produced by the $\mathrm{PMH}$ system can reductively decompose organic pollutants with oxidative nature (e.g., refractory azo dyes) under anaerobic circumstances. Moreover, based on the redox reactions occurring on the surface of photocatalysts and the enzymatic reactions in microbes, the PMH system can convert the valences of multiple heavy metal ions into less toxic or even nontoxic status simultaneously. In this review, we introduce the recent advances of using the $\mathrm{PMH}$ system in treating environmental pollutions and compare this system with another similar system, the traditional intimately coupled photocatalysis and biodegradation (ICPB) system. Finally, the current challenges and future directions in this field are discussed as well.

Keywords: photocatalytic material, microbe, hybrid, environmental remediation, solar power

\section{INTRODUCTION}

Environmental pollution is one of the world's most severe problems that we have to face nowadays. This has caused great concern globally, and it is urgent to develop more efficient methods to handle environmental issues. Photocatalytic oxidation and microbe bioremediation are considered as two promising methods and have been extensively explored (Lin et al., 2012; Karim et al., 2021). Photocatalytic oxidation-based approaches mainly utilize the photogenerated holes and electrons on the surfaces of photocatalytic materials to generate reactive oxygen species (ROS), which will then oxidatively decompose pollutants (Byrne et al., 2018). Photocatalytic oxidation provides the possibility of utilizing sustainable and green solar energy to remove pollutants. As for microbe- 
based bioremediation, pollutants are removed by the versatile catabolic processes in microbes, and thereby, this method is costeffective and eco-friendly (Juan-Luis et al., 2011).

Recently, photocatalytic materials and microbes are intimately combined together to form the so-called photocatalytic material-microbe hybrid (PMH) system (Du et al., 2020). In 2016, the PMH system is firstly built by Professor Peidong Yang' group in Berkeley, University of California, to realize solar-tochemical production (Sakimoto et al., 2016). The PMH system combines the advantages of abiotic catalysis and biotransformation for the production of value-added chemicals and fuels. Photocatalytic materials sustainably provide reducing power from light to microbe, which then uses these reducing equivalents to synthesize products. Currently, $\mathrm{PMH}$ systems are mainly applied for $\mathrm{H}_{2}$ evolution (Jiang et al., 2018; Wei et al., 2018), $\mathrm{CO}_{2}$ conversion (Ye et al., 2019), $\mathrm{N}_{2}$ fixation (Wang $\mathrm{B}$. et al., 2019; Chen et al., 2019), and chemical manufacturing (Guo et al., 2018; Zhang et al., 2020).

Interestingly, this PMH system has also shown great potential in treating environmental pollutants in some very recent reports (Wang X. et al., 2019). Yet, to the best of our knowledge, there is no review summarizing these advances. Therefore, we summarize the current advances of using the PMH system in environmental remediation and compare it with another similar system, the intimately coupled photocatalysis and biodegradation (ICPB). Current research gaps and future perspectives are also given in the end. We believe this review will be helpful for the future development of this direction.

\section{APPLICATION OF PMHS IN ENVIRONMENTAL REMEDIATION}

Most organic contaminants can be gradually biodegraded and completely mineralized through the aerobic respiration of microbes. Yet, the conventional aerobic treatment increases the energy consumption because of the high aeration rate during the operation process (Mitsugu and Yasumoto, 2003). In addition, some pollutants with oxidative nature (such as refractory azo dyes and halogenated organic and nitroaromatic compounds) tend to be degraded reductively rather than oxidatively (Liu P.-C. et al., 2020). Therefore, it is meaningful to develop new technologies which can provide adequate reducing power to fulfill the reduction degradation of these pollutants under anaerobic conditions. In this context, Xiao et al. (2019) coupled an electrochemically active bacterium, Shewanella oneidensis MR-1, with the $\mathrm{Ag}_{3} \mathrm{PO}_{4}$ photocatalyst to build a PMH system, which can remove almost $100 \%$ of $15 \mathrm{mg} / \mathrm{L}$ rhodamine B after 7 days under visible light irradiation. Blocking the Mtr respiratory pathway (a transmembrane electron transport chain) or dosing additional riboflavin (an electron shuttle) could interfere with the extracellular electron transfer of S. oneidensis MR-1 and decrease the degradation rate of rhodamine $\mathrm{B}$. The authors believed that the photogenerated holes on the $\mathrm{Ag}_{3} \mathrm{PO}_{4}$ photocatalyst were scavenged by the electrons released from S. oneidensis MR-1, and thereby, the photogenerated electrons on the $\mathrm{Ag}_{3} \mathrm{PO}_{4}$ photocatalyst were efficiently transferred to rhodamine $\mathrm{B}$ and achieve the photoreductive degradation (Figure 1A). Xiao et al. also used this $\mathrm{PMH}$ system to reductively decompose $p$-chlorophenol under anaerobic circumstances. The results of density functional theory calculations further proved that the electrochemically active bacterium S. oneidensis MR-1 could act as a biological hole scavenger (Liu P.-C. et al., 2020). In another of their work, they used CdS nanocrystals instead of $\mathrm{Ag}_{3} \mathrm{PO}_{4}$ to combine with S. oneidensis MR-1 and form a PMH system for the treatment of trypan blue (Xiao et al., 2020). Again, the biogenic electrons produced by the anaerobic respiration of S. oneidensis MR-1 eliminated the photogenerated holes on CdS nanocrystals and maintained the ongoing photoexcitation to produce electrons, cleaving the azo bonds in trypan blue.

Similarly, to degrade another azo dye, methyl orange (MO), Huang et al. (2019) built a PMH system by precipitating CdS nanoparticles on the surface of Geobacter sulfurreducens (an electrochemically active bacterium). This $\mathrm{PMH}$ system can completely degrade $40 \mathrm{mg} / \mathrm{L}$ MO after $3 \mathrm{~h}$ under light irradiation. Different from the mechanism proposed by Liu et al. (2020b) and Xiao et al. (2019, 2020), Huang et al. believed that there were two degradation pathways working simultaneously in their PMH system (Figure 1B): (1) the photogenerated electrons were stored on the outer-membrane cytochrome $\mathrm{C}$ of G. sulfurreducens, and these electrons were then transferred to the terminal reductase for mineralizing $\mathrm{MO}$; and (2) the photogenerated electrons could be directly transferred to the MO and degrade it. Huang et al. (2019) hypothesized that the photogenerated holes were scavenged by the intermediates produced during the decolorization process of $\mathrm{MO}$ as they found that dosing additional sacrificial reagents in the $\mathrm{PMH}$ system did not affect the MO degradation rate. In the report contributed by Chen et al. (2019), a PMH system containing CdS nanomaterials and Thiobacillus denitrificans was used to supply electrons for the denitrification process. After $68 \mathrm{~h}$ of visible light irradiation, this $\mathrm{PMH}$ system converted more than $72.1 \%$ of the $\mathrm{NO}_{3}{ }^{-}-\mathrm{N}$ into $\mathrm{N}_{2} \mathrm{O}-\mathrm{N}$. Lactate was chosen as the sacrificial reagent to scavenge the photogenerated holes. The authors proposed that the excited photoelectrons from CdS nanomaterials were transferred into microbes through the electron acceptors in cell membranes like C-type cytochrome and then to those enzymes involved in the denitrification pathway (Figure 1C).

Apart from persistent organic pollutants, the $\mathrm{PMH}$ system can also be used to treat heavy metal contamination. Our group deposited CdS nanomaterials on the surface of Bacillus thuringiensis HM-311 (a multiple-heavy-metal-tolerant strain isolated from heavy-metal-polluted soil (Berrazeg et al., 2019)) to form the PMH system (Zuo et al., 2021b). Then this PMH system was operated in the simulated $\mathrm{Cr}^{6+}(100 \mathrm{mg} / \mathrm{L})$ and $\mathrm{As}^{3+}$ $(100 \mathrm{mg} / \mathrm{L})$ co-contaminated water under visible light. After $24 \mathrm{~h}$ of operation, $99.25 \%$ of $\mathrm{Cr}^{6+}$ was reduced. Meanwhile, $\mathrm{As}^{3+}$ was simultaneously oxidized into $\mathrm{As}^{5+}$ by this $\mathrm{PMH}$ system, and up to $62.74 \mathrm{mg} / \mathrm{L}$ of $\mathrm{As}^{5+}$ was detected. We believed that with the help of solar energy and microbial enzymatic reactions, electrons can be transferred from heavy metal ions that need to be oxidized to detoxify them and donated to heavy metal ions that need to be reduced to become less toxic (Figures 1D,E). This PMH system 


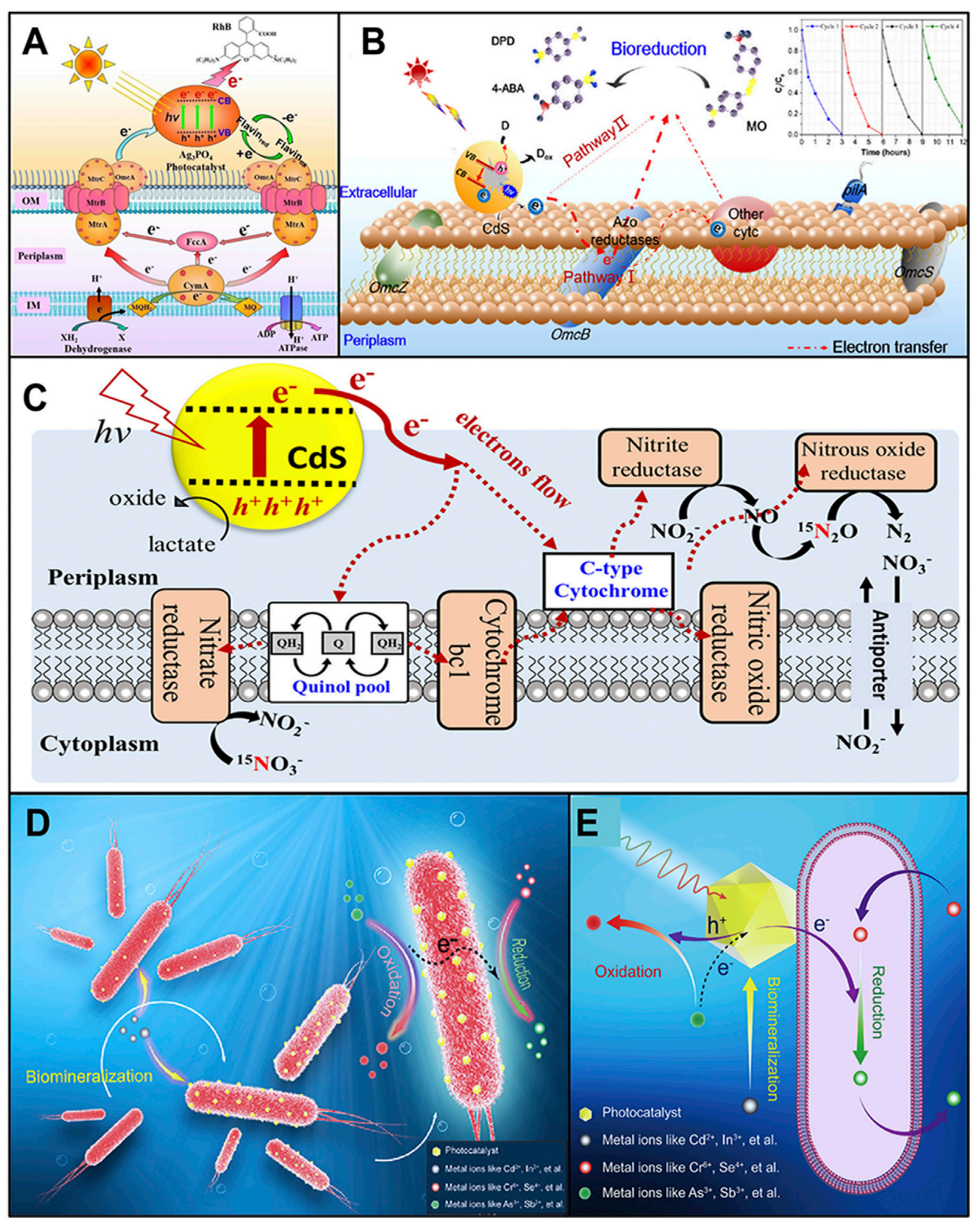

FIGURE 1 | (A) Schematic illustration of the light-driven degradation pathway of rhodamine B using the $\mathrm{Ag}_{3} \mathrm{PO}_{4}-\mathrm{S}$. oneidensis MR-1 hybrid system (reprinted with permission from Xiao et al., 2019). (B) Schematic illustration of the degradation pathway of MO using the CdS-G. sulfurreducens hybrid system (reprinted with permission from Huang et al., 2019). (C) Schematic illustration of the denitrification mechanism in the CdS-T. denitrificans hybrid system driven by light (reprinted with permission from Chen et al., 2019). (D, E) Mechanism of fabricating the CdS nanomaterial-B. thuringiensis HM-311 hybrid based on the microbial mineralization ability (D) and the electron transfer between different heavy metal ions in the hybrid system (E) (reprinted with permission from Zuo et al., 2021a). 
might realize the electron transfer between different heavy metal ions, achieving the repair of multiple metal ions in wastewater simultaneously.

\section{COMPARE PMH WITH ICPB}

In fact, it is not the first time that photocatalysts are combined with microbes intimately for environmental remediation. The group led by Professor Rittmann at Arizona State University first built a system which intimately coupled photocatalytic oxidation with microbial degradation (ICPB) for wastewater treatment in 2008 (Marsolek et al., 2010). Herein, we compare the ICPB system with the PMH system. This will help researchers understand the differences and connections between these two systems and apply them more properly for wastewater treatment.

\section{Mechanisms and Target Pollutants}

A typical ICPB system mainly consists of porous carriers, photocatalysts, biofilms, and an illuminated reactor. Photocatalysts are loaded on the surface of the carriers, and the biofilms are cultivated in the carriers. The porous carrier can adsorb refractory pollutants from the solution and enhance its mass transfer to the surface of the photocatalysts. In the process of photocatalytic degradation, a photocatalyst absorbs photon energy and generates electron-hole pairs, which will cause ROS such as $\bullet \mathrm{OH}$ and $\bullet \mathrm{O}_{2}{ }^{-}$. These ROS will attack and destroy the refractory organic contaminants and convert them into biodegradable products, which are completely degraded by the biofilms afterwards (Liu J. et al., 2020; Wu et al., 2020). ICPB systems are suitable to mineralize most of the refractory organic pollutants (e.g., antibiotics, dyes, and polycyclic aromatic hydrocarbons). A typical $\mathrm{PMH}$ system is mainly composed of microbial cells and photocatalytic materials deposited directly on their surfaces. For environmental remediation, electrochemically active bacteria are often used in $\mathrm{PMH}$ systems to provide the biogenic electrons to eliminate the photogenerated holes on the photocatalysts. In this case, the photogenerated electrons are able to reductively degrade those organic pollutants with oxidative natures (such as nitroaromatic compounds and azo dyes). In the PMH system for treating heavy metals, electrons generated by photocatalysts can be transferred into microbial cells to promote intracellular enzymatic reductions, reducing heavy metal ions such as $\mathrm{Cr}^{6+}$ and $\mathrm{Se}^{4+}$. Heavy metal ions like $\mathrm{As}^{3+}$ and $\mathrm{Sb}^{3+}$ can be oxidized by photogenerated holes as sacrificial electron donors.

\section{Light Source}

In the PMH system, additional carriers are not required as photocatalysts are directly coated on the surface of bacteria. Considering that most microbes cannot survive under ultraviolet (UV) light irradiation, the $\mathrm{PMH}$ system is often driven by visible light. For the ICPB system, the excitation light can be UV light or visible light since the biofilms are protected by the carriers. Yet, we have to point out that although UV-light-excited photocatalysts display good efficiencies in ICPB, their practical applications are still not widespread because UV light accounts for only 4\% of sunlight (Zhang et al., 2016). Plus, UV light exhibits a strong killing ability on microorganisms. Under UV light irradiation, biofilms are detached from the carriers and the soluble microbial products are generated, which increase the turbidity of the reaction mixture. This could in turn hinder the penetration of UV light and thereby reduce the photocatalytic degradation efficiency (Zuo et al., 2021a). Therefore, most ICPB systems are driven by visible light now.

\section{Microbes}

In the ICPB system, microbes are the implementers of biodegradation. It could be single strain with the ability to degrade the products from the photooxidation stage. Yet, more ICPB systems choose the microbial consortium as the implementers of biodegradation because it is hard to completely degrade organic pollutants by one microbial strain. Microbial consortium contains different types of microorganisms, can corporate with each other, and show a synergy effect when they degrade organic pollutants. Thus, the microbial consortium can degrade different types of organic pollutants and their toxic intermediate metabolites, achieving a complete-degradation effect. In the PMH systems designed for removing organic pollutants, electrochemically active bacteria are usually chosen. These bacteria can provide bio-electrons via their anaerobic respiration to scavenge the photogenerated holes on the photocatalysts and maintain the ongoing photoreductive degradation of those oxidative organic pollutants. These bacteria are not capable of decomposing the intermediates produced from the photoreductive degradation process.

\section{Electron Donors}

For ICPB systems, microbes are key degraders which completely mineralize the photocatalysis products. However, this biodegradation process could be compromised if the photocatalysis products remain refractory or inhibitory. Providing readily biodegradable co-substrates such as acetate which could act as extra electron donors and energy sources will improve the microbial metabolic activity, counteract toxicity effects, and accelerate oxygenation reactions, resulting in a great increase of the removal rate (Bai et al., 2015; Xiong et al., 2018). In PMH systems, photogenerated holes need to be scavenged, which will ensure the system will provide reductive electrons for microbial synthesis or the photoreductive degradation of target pollutants. For those PMH systems used for environmental purposes, the hole scavengers are the electrochemically active bacteria, which need carbon sources and electron donors such as lactate and sodium acetate trihydrate. In our previous work, some heavy metal ions (e.g., $\mathrm{As}^{3+}$ and $\mathrm{Sb}^{3+}$ ) can be used as sacrificial electron donors to maintain the continuous operation of the PMH system (Zuo et al., 2021b).

\section{Operation Conditions}

As shown in Figure 2, the ICPB system is agitated and operated in an illuminated reactor coupled with an aeration pump, which supplies enough oxygen for the photocatalysis oxidation and microbial respiration. In contrast, $\mathrm{PMH}$ systems can work 


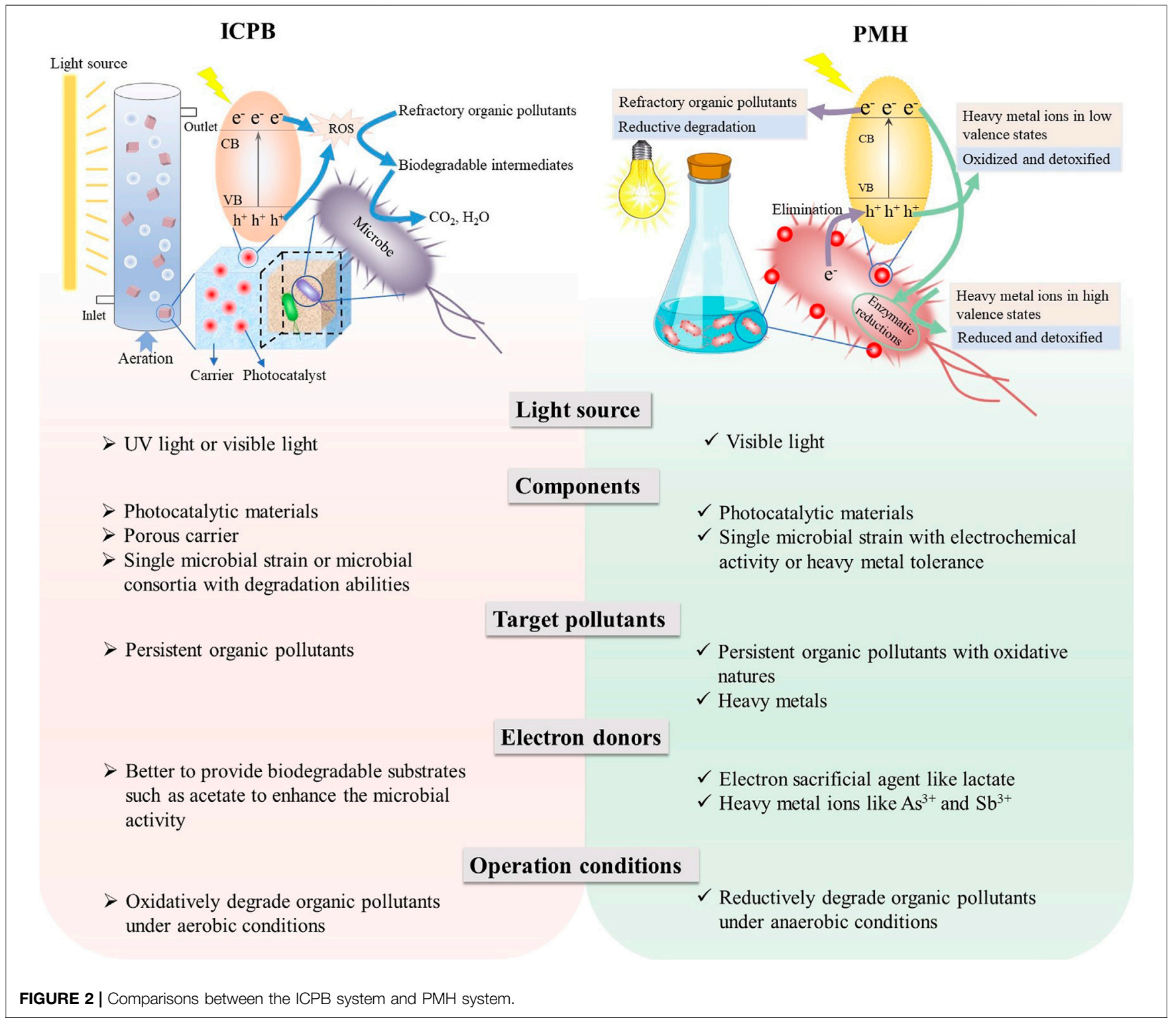

under anaerobic conditions to achieve photoreductive degradation of those oxidative organic pollutants. Since it does not need to aerate, the PMH system decreases the energy consumption during the operation process.

\section{CONCLUSIONS AND PERSPECTIVE}

Herein, we summarize the recent advances of using the $\mathrm{PMH}$ systems for environmental remediations. Driven by sustainable, green sunlight, these emerging $\mathrm{PMH}$ systems may provide new and promising alternatives for anaerobically photoreductive decomposing organic pollutants with oxidative natures and repairing multiple heavy metal ions simultaneously in wastewater. While some exciting results have already been obtained, the PMH system is still in its infancy, and some gaps that need more effort to address remain:
1) The photocatalytic materials used in $\mathrm{PMH}$ systems now often contain heavy metals or noble metals (e.g., CdS and $\mathrm{Ag}_{3} \mathrm{PO}_{4}$ ); more investigations are needed to address their biocompatibility, cost, and environmental impacts before implementing real environmental applications. Interestingly, some microbes can utilize natural extracellular semiconducting minerals (e.g., iron and manganese) to synthesize photosensitizers, which can decorate microbial membranes, facilitate the transmembrane electron transfer, and achieve light harvesting (Lu et al., 2019; Yu and Leadbetter, 2020). Thus, it might be a promising direction to synthesize earthabundant semiconductor minerals as compatible photosensitizers for PMH systems based on the microbes' biomineralization capabilities.

2) Microorganisms are the other key component in the $\mathrm{PMH}$ system. It is critical to improve the microbes' capability to yield and transfer electrons, which requires us to discover and 
redesign the corresponding molecular pathways rationally in the future. The incorporation of more biology scientists especially with omics and synthetic biology backgrounds will be great.

3) Electron donors, either for the microbial growth or for the scavenging of the photogenerated holes on the photocatalysts directly, are crucial for the continuous operation of $\mathrm{PMH}$ systems. The costs of those typical hole scavengers such as cysteine, ascorbic acid, and HEPES are too high for large-scale application. Finding electron donors that can be regenerated and have low cost, as well as being biocompatible, will be of great benefit to ensure economic viability and applicability of $\mathrm{PMH}$ systems in environmental applications.

\section{REFERENCES}

Bai, Q., Yang, L., Li, R., Chen, B., Zhang, L., Zhang, Y., et al. (2015). Accelerating Quinoline Biodegradation and Oxidation with Endogenous Electron Donors. Environ. Sci. Technol. 49 (19), 11536-11542. doi:10.1021/acs.est.5b03293

Berrazeg, M., Deriet, A., De Keersmaecker, S. C. J., Verhaegen, B., Vanneste, K., Botteldoorn, N., et al. (2019). Whole-Genome Sequencing of MultidrugResistant Escherichia coli Strains Harboring the Mcr-1 Gene, Isolated from Seawater of the Algiers Coast in Algeria. Microbiol. Resour. Announc 8 (34), e00638-19. doi:10.1128/MRA.00638-19

Byrne, C., Subramanian, G., and Pillai, S. C. (2018). Recent Advances in Photocatalysis for Environmental Applications. J. Environ. Chem. Eng. 6 (3), 3531-3555. doi:10.1016/j.jece.2017.07.080

Chen, M., Zhou, X.-F., Yu, Y.-Q., Liu, X., Zeng, R. J.-X., Zhou, S.-G., et al. (2019). Light-driven Nitrous Oxide Production via Autotrophic Denitrification by SelfPhotosensitized Thiobacillus Denitrificans. Environ. Int. 127, 353-360. doi:10.1016/j.envint.2019.03.045

Du, Y., Guo, J., Chen, Z., Song, Y., Lu, C., Han, Y., et al. (2020). A New Solar-Driven Biological Wastewater Treatment Technology Based on the Synergy of Photosensitizers and Non-photosynthetic Bacteria. Int. Biodeterioration Biodegradation 155, 105111. doi:10.1016/j.ibiod.2020.105111

Guo, J., Suástegui, M., Sakimoto, K. K., Moody, V. M., Xiao, G., Nocera, D. G., et al. (2018). Light-driven fine Chemical Production in Yeast Biohybrids. Science 362 (6416), 813-816. doi:10.1126/science.aat9777

Huang, S., Tang, J., Liu, X., Dong, G., and Zhou, S. (2019). Fast Light-Driven Biodecolorization by a Geobacter Sulfurreducens-Cds Biohybrid. ACS Sustain. Chem. Eng. 7 (18), 15427-15433. doi:10.1021/ acssuschemeng.9b02870

Jiang, Z., Wang, B., Yu, J. C., Wang, J., An, T., Zhao, H., et al. (2018). AglnS2/In2S3 Heterostructure Sensitization of Escherichia coli for Sustainable Hydrogen Production. Nano Energy 46, 234-240. doi:10.1016/j.nanoen.2018.02.001

Karim, A., Islam, M. A., Khalid, Z. B., Yousuf, A., Khan, M. M. R., and Mohammad Faizal, C. K. (2021). Microbial Lipid Accumulation through Bioremediation of palm Oil Mill Effluent Using a Yeast-Bacteria Co-culture. Renew. Energ. 176, 106-114. doi:10.1016/j.renene.2021.05.055

Lin, F., Zhang, Y., Wang, L., Zhang, Y., Wang, D., Yang, M., et al. (2012). Highly Efficient Photocatalytic Oxidation of Sulfur-Containing Organic Compounds and Dyes on Tio2 with Dual Cocatalysts Pt and Ruo2. Appl. Catal. B: Environ. 127, 363-370. doi:10.1016/j.apcatb.2012.08.024

Liu, J., Luo, Z., Han, W., Zhao, Y., and Li, P. (2020a). Preparation of ZnO/ Bi2WO6 Heterostructures with Improved Photocatalytic Performance. Mater. Sci. Semiconductor Process. 106, 104761. doi:10.1016/ j.mssp.2019.104761

Liu, P.-C., Ma, X.-L., Li, T.-T., Yan, F., Wu, L.-J., and Xiao, X. (2020b). Elucidation of Photodegradation of P-Chlorophenol in a Biophotoelectric Reductive Degradation System by Density Functional Theory Calculations. Int. Biodeterioration Biodegradation 151, 104969. doi:10.1016/ j.ibiod.2020.104969

Lu, A., Li, Y., Ding, H., Xu, X., Li, Y., Ren, G., et al. (2019). Photoelectric Conversion on Earth's Surface via Widespread Fe- and Mn-mineral Coatings.

\section{AUTHOR CONTRIBUTIONS}

YY, SW, and JT contributed to the figure and manuscript draft preparations. AZ, SG, XT, and HL revised the manuscript. All authors approved it for publication.

\section{FUNDING}

This work was supported by the Open Project of State Key Laboratory of Urban Water Resource and Environment (Grant No. HC202147) and Slovenian Research Agency (ARRS: P40165).

Proc. Natl. Acad. Sci. USA 116 (20), 9741-9746. doi:10.1073/ pnas. 1902473116

Marsolek, M. D., Torres, C. I., Hausner, M., and Rittmann, B. E. (2008). Intimate Coupling of Photocatalysis and Biodegradation in a Photocatalytic Circulating-Bed Biofilm Reactor. Biotechnol. Bioeng. 101 (1), 83-92. doi:10.1002/bit.21889

Ramos, J.-L., Marqués, S., van Dillewijn, P., Espinosa-Urgel, M., Segura, A., Duque, E., et al. (2011). Laboratory Research Aimed at Closing the Gaps in Microbial Bioremediation. Trends Biotechnol. 29 (12), 641-647. doi:10.1016/ j.tibtech.2011.06.007

Saito, M., and Magara, Y. (2003). Removal of Organic Pollutants and Metabolic Adaptation of Microorganisms by Micro-aeration. J. Environ. Sci. Health A 38 (6), 991-1005. doi:10.1081/ESE-120019858

Sakimoto, K. K., Wong, A. B., and Yang, P. (2016). Self-photosensitization of Nonphotosynthetic Bacteria for Solar-To-Chemical Production. Science 351, 74-77. doi:10.1126/science.aad3317

Wang, B., Xiao, K., Jiang, Z., Wang, J., Yu, J. C., and Wong, P. K. (2019a). Biohybrid Photoheterotrophic Metabolism for Significant Enhancement of Biological Nitrogen Fixation in Pure Microbial Cultures. Energy Environ. Sci. 12 (7), 2185-2191. doi:10.1039/C9EE00705A

Wang, X., Pu, J., Liu, Y., Ba, F., Cui, M., Li, K., et al. (2019b). Immobilization of Functional Nano-Objects in Living Engineered Bacterial Biofilms for Catalytic Applications. Natl. Sci. Rev. 6 (5), 929-943. doi:10.1093/nsr/ nwz104

Wei, W., Sun, P., Li, Z., Song, K., Su, W., Wang, B., et al. (2018). A Surface-Display Biohybrid Approach to Light-Driven Hydrogen Production in Air. Sci. Adv. 4 (2), eaap9253. doi:10.1126/sciadv.aap9253

Wu, Y., Zhong, L., Yuan, J., Xiang, W., Xin, X., Liu, H., et al. (2020). Photocatalytic Optical Fibers for Degradation of Organic Pollutants in Wastewater: a Review. Environ. Chem. Lett. 19 (70), 1335-1346. doi:10.1007/s10311-020-01141-3

Xiao, X., Han, X., Wang, L.-G., Long, F., Ma, X.-L., Xu, C.-C., et al. (2020). Anaerobically Photoreductive Degradation by Cds Nanocrystal: Biofabrication Process and Bioelectron-Driven Reaction Coupled with Shewanella Oneidensis MR-1. Biochem. Eng. J. 154 (15), 107466. doi:10.1016/j.bej.2019.107466

Xiao, X., Ma, X.-L., Liu, Z.-Y., Li, W.-W., Yuan, H., Ma, X.-B., et al. (2019). Degradation of Rhodamine B in a Novel Bio-Photoelectric Reductive System Composed of Shewanella Oneidensis Mr-1 and Ag3PO4. Environ. Int. 126, 560-567. doi:10.1016/j.envint.2019.03.010

Xiong, H., Dong, S., Zhang, J., Zhou, D., and Rittmann, B. E. (2018). Roles of an Easily Biodegradable Co-substrate in Enhancing Tetracycline Treatment in an Intimately Coupled Photocatalytic-Biological Reactor. Water Res. 136, 75-83. doi:10.1016/j.watres.2018.02.061

Ye, J., Yu, J., Zhang, Y., Chen, M., Liu, X., Zhou, S., et al. (2019). Light-driven Carbon Dioxide Reduction to Methane by Methanosarcina Barkeri-CdS Biohybrid. Appl. Catal. B: Environ. 257 (15), 117916. doi:10.1016/ j.apcatb.2019.117916

Yu, H., and Leadbetter, J. R. (2020). Bacterial Chemolithoautotrophy via Manganese Oxidation. Nature 583 (7816), 453-458. doi:10.1038/s41586-0202468-5 
Zhang, L., Xing, Z., Zhang, H., Li, Z., Wu, X., Zhang, X., et al. (2016). High Thermostable Ordered Mesoporous SiO2-TiO2 Coated Circulating-Bed Biofilm Reactor for Unpredictable Photocatalytic and Biocatalytic Performance. Appl. Catal. B: Environ. 180, 521-529. doi:10.1016/ j.apcatb.2015.07.002

Zhang, R., He, Y., Yi, J., Zhang, L., Shen, C., Liu, S., et al. (2020). Proteomic and Metabolic Elucidation of Solar-Powered Biomanufacturing by BioAbiotic Hybrid System. chem 6 (1), 234-249. doi:10.1016/ j.chempr.2019.11.002

Zuo, W., Yu, Y., and Huang, H. (2021a). Making Waves: MicrobePhotocatalyst Hybrids May Provide New Opportunities for Treating Heavy Metal Polluted Wastewater. Water Res. 195, 116984. doi:10.1016/ j.watres.2021.116984

Zuo, W., Zhang, L., Zhang, Z., Tang, S., Sun, Y., Huang, H., et al. (2021b). Degradation of Organic Pollutants by Intimately Coupling Photocatalytic Materials with Microbes: a Review. Crit. Rev. Biotechnol. 41 (6), 273-299. doi:10.1080/07388551.2020.1869689
Conflict of Interest: The authors declare that the research was conducted in the absence of any commercial or financial relationships that could be construed as a potential conflict of interest.

Publisher's Note: All claims expressed in this article are solely those of the authors and do not necessarily represent those of their affiliated organizations, or those of the publisher, the editors and the reviewers. Any product that may be evaluated in this article, or claim that may be made by its manufacturer, is not guaranteed or endorsed by the publisher.

Copyright $\odot 2022 \mathrm{Yu}$, Wang, Teng, Zupanic, Guo, Tang and Liang. This is an openaccess article distributed under the terms of the Creative Commons Attribution License (CC BY). The use, distribution or reproduction in other forums is permitted, provided the original author(s) and the copyright owner(s) are credited and that the original publication in this journal is cited, in accordance with accepted academic practice. No use, distribution or reproduction is permitted which does not comply with these terms. 